\title{
Electrical Impedance Tomography as a Primary Screening Technique for Breast Cancer Detection
}

\author{
Mehrnoosh Akhtari-Zavare ${ }^{1}$, Latiffah A Latiff ${ }^{1,2 *}$
}

\begin{abstract}
Electrical impedance tomography (EIT) is a new non-invasive, mobile screening method which does not use ionizing radiation to the human breast. It is based on the theory that cancer cells display altered local dielectric properties, thus demonstrating measurably higher conductivity values. This article reviews the utilisation of EIT in breast cancer detection. It could be used as an adjunct to mammography and ultrasonography for breast cancer screening.
\end{abstract}

Keywords: Breast cancer - electro-impedance tomography - electrical impedance mammography

Asian Pac J Cancer Prev, 16 (14), 5595-5597

\section{Introduction}

Breast cancer is the most common cancer among women and leading cause of death worldwide, including Malaysia (Loh et al., 2011; Kulakci et al., 2015). The breast cancer accounts for $30-40 \%$ of all the cancers in women all over the world (Che et al., 2014; Kulakci et al., 2015). In Malaysia, about one in 19 women in this country are at risk for getting breast cancer, compared to one in eight in Europe and the United States (National Cancer Registry, 2006). According to World Health Organization, more than $30 \%$ of cancer deaths can be prevented (World Health Organization, 2009). Hence, the best way to put a stop to death is by early detection when treatment is more effective (Myoung et al., 2007).

There are two strategies for early detection of breast cancer: $i$ ) education on recognizing early signs of cancer and seeking prompt medical attention. ii) screening programmes to identify early cancer or pre-cancer stage before signs are recognizable (Ministry of Health Malaysia, 2009).

Currently, instrumental methods of breast disease diagnostics can be divided into two groups. To the first group belong X-ray mammography (MMG) and ultrasonography (USG). These methods are based on a study of the mammary gland anatomy. They determine the presence or absence of the pathology in the organ's structure (Renata et al., 2012). Result of study done by Chang et al. (2015) showed breast cancer survival rate in Korea has increased consistently due to the increasing using mammography among women.

To the second group belong diagnostic methods, based on physiology, on the peculiarities of metabolism in breast tissues in norm and pathology. These methods include electrical impedance tomography (EIT) (Renata et al., 2012).

As mention earlier, mammography is a method used for early detection of breast cancer. But, mammographic facilities are costly and available only at limited places (Vithana et al., 2015). Consequently, the new technology electrical impedance tomography (EIT) which is a relatively new imaging method that has evolved over the past 20 years is recommended (Hope et al., 2004). Electrical Impedance Computerized Mammograph MEIK" is an EIT system for breast diagnostics which has been approved for clinical usage in Russia.

Electrical impedance tomography (EIT) which is a noninvasive procedure using electrical impedance to image the human breast (Chakraborti et al., 2010). This scanning device does not emit any ionizing radiation thus it can be done on pregnant women by means of no age limit and not expensive (Kinouchi et al., 2002). Due to its mobility and using non compression technique it is appealing to patients.

The value of EIT is based on the diverse electrical storage potential of normal and pathologically changed tissues. These differences will allow to image differences in the tissue conductivity and permittivity inferred from the breast tissue electrical measurements. It consists of a handheld transducer and a computer screen that displays the images of the breast. Small currents are applied to a part of the subject's body through conducting electrodes attached to the skin and the resulting electrical potentials are measured and visualized (Renata et al., 2012).

As mention earlier, the MEIK device, using EIT technology, is radiation free, and since it is portable, the screening can be done virtually anywhere. This handheld device consists of a compact array of 256 electrodes 
Table 1. HPE Findings Comparative Analysis

\begin{tabular}{|c|c|c|c|}
\hline \multirow{2}{*}{ HPE* $^{*}$} & \multicolumn{2}{|c|}{ Sensitivity } \\
\hline \multirow{2}{*}{ Fibrocystic mastitis } & $\begin{array}{c}\text { Mammography } \\
(\mathrm{MG})\end{array}$ & $\begin{array}{c}\text { Ultrasonography } \\
\text { (USG) }\end{array}$ & $\begin{array}{c}\text { 3D Electrical Impedance } \\
\text { Tomography }\end{array}$ \\
\cline { 2 - 4 } & $83.3 \%$, & $94.4 \%$, & $77.8 \%$, \\
\hline \multirow{2}{*}{ Cyst } & $95 \%$ CI 58.6\% to $96.4 \%$ & $95 \%$ CI $72.7 \%$ to $99.9 \%$ & $95 \%$ CI $52.4 \%$ to $93.4 \%$ \\
\cline { 2 - 4 } & $95 \%$ CI $58.1 \%$ to $94.6 \%$ & $95 \%$ CI $83.9 \%$ to $100 \%$ & $95 \%$ CI $83.9 \%$ to $100 \%$ \\
\hline \multirow{2}{*}{ Fibroadenoma } & $87.5 \%$, & $75.0 \%$, & $68.8 \%$, \\
\cline { 2 - 4 } & $95 \%$ CI $61.7 \%$ to $98.5 \%$ & $95 \%$ CI $57.2 \%$ to $92.7 \%$ & $95 \%$ CI $41.3 \%$ to $88.9 \%$ \\
\hline \multirow{2}{*}{ Carcinoma } & $100 \%$, & $100 \%$, & $75.0 \%$, \\
\cline { 2 - 4 } & $95 \%$ CI 39.8\% to $100 \%$ & $95 \%$ CI $39.8 \%$ to $100 \%$ & $95 \%$ CI $19.4 \%$ to $99.4 \%$ \\
\hline
\end{tabular}

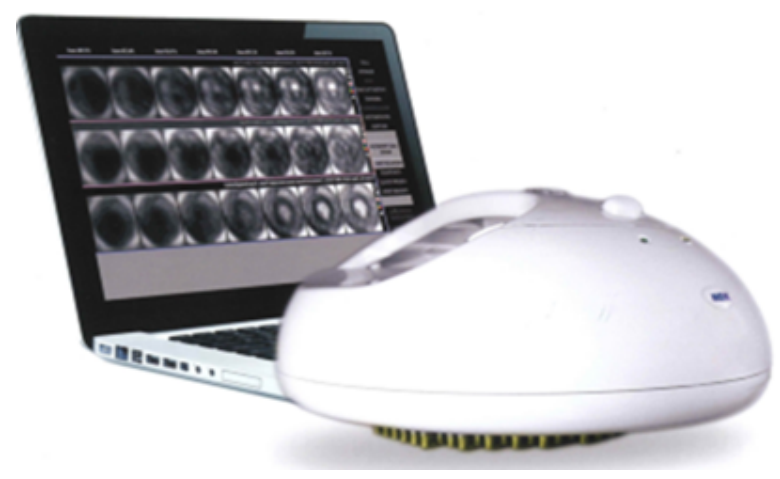

Figure 1. Electrical Impedance Computer Mammography

and uses small electrical pulses $(0.5 \mathrm{~mA}, 50 \mathrm{kHz})$ for the breast examination. The procedure continues for each electrode until 65,280 voltage measurements are taken within 20 seconds. The received data is reconstructed into direct-to-digital tomography conductivity images of the breast. Seven tomography slices are then created within 40 seconds at the depths of $0.4 \mathrm{~cm}$ to $4.6 \mathrm{~cm}$ to detect and isolate tumours. No materials like gel or ECG spray are required for the procedure (Ministry of Health, 2009) (Figure 1).

Experimental studies with Electro Impedance Tomography (EIT) have shown that significant changes occur in the electrical properties of breast cancer tissue compared to the surronding normal tissue (Martin et al., 2002; Zou et al., 2003). Some evidence has been found that malignant breast tumours have lower electrical impedance than surrounding normal tissues (Malich et al., 2001; Prasad et al., 2008). This observation has led to the proposal that electrical impedance could be used as an indicator for breast cancer detection (Cherepenin et al., 2001; Cherepenin et al., 2002; Athanasiou et al., 2007).

\section{Methodology}

Four databased were used for the literature search Pub Med, Medline, Cochrane, INAHTA. The search terms were, "breast cancer", "Electro- Impedance Tomography" and "electrical impedance mammography". Any primary and secondary papers pertaining to "Electrical Impedance Tomography" or "Electrical Impedance Mammography" included in this technology review. Those which full text could not be obtained were excluded.

\section{Effectiveness of EIT}

A cross-sectional study was conducted to determine the diagnostic efficiency of 3D Electrical Impedance Tomography (EIT) as compared to Mammography (MG) and Ultrasonography (USG) in imaging the breast (Prasad et al., 2008). A total of 88 patients presenting with various breast complaints was examined using MG, USG and 3D EIT imaging system " $M E I K$ ". The sensitivity of 3D EIT in diagnosing different breast pathology was found to be less sensitive compared to mammography and ultrasonography (Prasad et al., 2008). Details of the analysis are shown in details of the analysis are shown in table 1.

The results of study showed that, no significant difference in sensitivity between mammography and ultrasonography $(\mathrm{p}=0.219)$, mammography and EIT $(\mathrm{p}=0.779)$, and ultrasonography and EIT $(\mathrm{p}=0.169)$. However, there was significant difference in the sensitivity of mammography compared to ultrasonography $(\mathrm{p}=0.042$ ) and EIT ( $\mathrm{p}=0.042)$ with regards to identifying cysts (Prasad et al., 2008).

In 2012, Raneta conducted a study among 808 patients on women from Slovakia found that the sensivity of EIT was $87 \%$, lower than MMG (89\%) and USG (91\%), but this differences is insignificant. The specificity of the EIT and USG showed almost equal values (85\% and $84 \%$, respectively) and specificity of MMG reached $91 \%$. Also, the findings of this study showed that, in combinations EIT + MMG and EIT + USG sensitivity increased to $96 \%$ and $98 \%$, respectively. The specificity of EIT + MMG amounted to $79 \%$ and of EIT+ USG to $71 \%$ (Raneta et al., 2012).

Zain et al. (2014) done one study among 150 symptomatic women underwent EIT examination in Department of Radiology, Universiti Kebangsaan Malaysia Medical Center, Malaysia. Results of this study showed EIT had $64.4 \%$ of sensitivity using diagnostic Mammography (MG), $70.1 \%$ of specificity, $67.1 \%$ of positive and $67.5 \%$ of negative predictive value. However, 
its specificity and positive predictive value increased from $70.1 \%$ to $76.3 \%$ and from $67.1 \%$ to $80 \%$ respectively when combination EIT, MG and USG were used (Zain et al., 2014). If the test results could not confirm the type of breast abnormality through MMG and US, additional biopsy procedure will take place. With respect to the histopathological findings (10 malignant and 13 benign lesions) 9 of 10 (90\%) malignant lesions were correctly identified whereas 10 of $13(76.9 \%)$ benign lesions were correctly identified as benign by EIT ( 3 of 13 benign lesions showed as false-positive findings) (Zain et al., 2014).

There are some factors which effect on mean electrical conductivity of EIT. For instance, in in study done by Zain et al. (2014a) showed that the mean electrical conductivity value of Malaysian women were increased with age, but this value is lower than the standard Caucasians $(\mathrm{p}<0.05)$. Also, their study showed there was strong positive correlation between ranked BMI and mean electrical conductivity value ( $\mathrm{rs}=0.28, \mathrm{p}=0.001)$.

\section{Conclusion}

In conclusion, based on the finding obtained from previous studies related to sensivity and specificity of EIT and comparing with mammography and ultrasound; it appropriate to use EIT for preventive check-ups of the female population between rounds of screening in order to isolate patients at risk; in examination of pregnant and lactating women and women with high risk of carcinogenesis (BRCA1, BRCA2 genes) and as an additional method

\section{References}

Athanasiou A, Vanel D, Fournier L, Balleyguier C (2007). Optical mammography: A new technique for visualizing breast lesions in women presenting non palpable BIRADS 4-5 imaging fi ndings: preliminary results with radiologicpathologic correlation. Cancer Imaging, 7, 34-40.

Chakraborti KL, Selvamurthy W (2010). Clinical application of electrical impedance tomography in the present health scenario of India. J Phys Conf Ser, 224, 12069.

Chang H L, Young IK (2015). Effects of attitude, social influence, and self-efficacy model factors on regular mammography performance in life transition aged women in Korea. Asian Pac J Cancer Prev, 16, 3429-34.

Che CC, Coomarasamy J, Suppayah DB (2014). Perception of breast health among malaysian female adolescents. Asian Pac J Cancer Prev, 15, 7175-80.

Cherepenin V, Karpov A, Korjenevsky A, et al (2001). A 3D electrical impedance tomography (EIT) system for breast cancer detection. Physiol Meas, 22, 9-18.

Cherepenin VA, Karpov AY, Korjenevsky AV, et al (2002). Three-dimensional EIT imaging of breast tissues: System design and clinical testing. IEEE Trans Med Imag, 21, 662-7.

Hope TA, Iles SE (2004). Technology review: the use of electrical impedance scanning in the detection of breast cancer. Breast Cancer Res, 6, 69-74.

Kinouchi Y, Pandya AS, Arimoto A, Agarwal A (2002). A novel approach for measuring electrical impedance tomography for local tissue with artificial intelligent algorithm. International J Biometrics Bioinformatics, 3, 66-81.
Kulakci H, Ayyildiz TK, Yildirim N, et al (2015). Effects of breast cancer fatalism on breast cancer awareness among nursing students in Turkey. Asian Pac J Cancer Prev, 16, 3565-72.

Loh SY, Chew SL (2011). Awareness and practice of breast self examination among Malaysian women with breast cancer. Asian Pac J Cancer Prev, 12, 199-202.

Malich A, Boehm T, Facius M, et al (2001). Differentiation of mammographically suspicious lesions: evaluation of breast ultrasound, MRI mammography and electrical impedance scanning as adjunctive technologies in breast cancer detection. Clin Radiol, 56, 278-283.

Martin G, Martin R, Brieva MJ, Santamaria L (2002). Electrical impedance scanning in breast cancer imaging: correlation with mammographic and histologic diagnosis. Eur Radiol, 12,1471-8.

National Cancer Registry (2006). Malaysian cancer statistics, data and figure peninsular Malaysia. ministry of health Malaysia, Malaysia.

Prasad SN, Houserkova D, Campbell J (2008). Breast imaging using 3D electrical impedance tomography. Biomed Pap Med Fac Univ Palacky Olomouc Czech Repub, 152, 151-4.

Renata O, Ondrus D, Bella V (2012). Utilisation of electrical impedance tomography in breast cancer diagnosis. Clin Oncol, 25, 36-41.

Vithana CH, Ariyaratne MAY, Jayawardana PL (2015). Effectiveness of an educational intervention among public health midwives on breast cancer early detection in the district of gampaha, sri lanka. Asian Pac J Cancer Prev, 16, 227-32.

World Health Organization (2009). Breast cancer: prevention and control [Online].

Zain NM, Chelliah KK (2014a). Breast imaging using electrical impedance tomography: correlation of quantitative assessment with visual interpretation. Asian Pac J Cancer Prev, 15, 1327-31

Zain NM, Kanaga KC, Sharifah MIA, Suraya A, Latar NH (2014). Study of electrical impedance tomography as a primary screening technique for breast cancer. IEEE conference on biomedical engineering and science, 8-10 Dec 2014, Miri, Sarawak, Malaysia.

Zou Y, Guo Z (2003). A review of electrical impedance techniques for breast cancer detection. Med Eng Phys, 25, 79-90. 